\title{
Present treatment options for atrial fibrillation
}

\author{
S K S Lairikyengbam, M H Anderson, A G Davies
}

Postgrad Med J 2003;79:67-73

Atrial fibrillation is the commonest sustained cardiac arrhythmia. It accounts for $>35 \%$ of all hospital admissions for cardiac arrhythmias in the United States. The presence of atrial fibrillation increases the mortality of a population by up to twofold. The risk of stroke increases from $1.5 \%$ in patients with atrial fibrillation from $50-59$ years of age to up to $23.5 \%$ for such patients aged 80-89 years. Although the diagnosis of atrial fibrillation is usually straightforward, effective treatment is not. This article will discuss how rhythm control of atrial fibrillation can best be achieved, the controversy over the rhythm versus rate control, the maintenance of sinus rhythm with antiarrhythmic drugs after cardioversion, and prevention of thromboembolism. Finally, the recent advances in various non-pharmacological approaches for the treatment of atrial fibrillation will be highlighted.

See end of article for authors' affiliations

Correspondence to: Dr S K S Lairikyengbam, Department of Medicine, Bronglais General Hospital, Aberystwyth SY23 1ER, UK: slairik@hotmail.com

Submitted 17 June 2002 Accepted

30 October 2002
A

trial fibrillation is the commonest cardiac arrhythmia. It affects $5 \%$ of British people above the age of 65 years and $10 \%$ above 75. ${ }^{1}$ It accounts for more than $35 \%$ of all hospital admissions for cardiac arrhythmias in the United States. ${ }^{2}$ Although thought to be immediately non-life threatening, its presence increases mortality by up to twofold. ${ }^{3}$ The most important morbidity and mortality associated with atrial fibrillation result from stroke. The attributable risk of stroke increased from $1.5 \%$ for patients with atrial fibrillation aged $50-59$ years to $23.5 \%$ for those aged 80-89 years. $^{4}$ It is also associated with congestive heart failure. These result in a significant increase in the of cost of health care in the community. Moreover, ischaemic stroke secondary to atrial fibrillation carries about twice the risk of death in comparison with stroke from other causes. ${ }^{5}$ Despite the high prevalence of atrial fibrillation and the increased morbidity and mortality associated with it, its treatment strategy remains less well defined. We review the literature and present here the evidence based treatment options for this most common sustained cardiac arrhythmia.

\section{DEFINITION}

Atrial fibrillation is defined when there is complete absence of coordinated atrial systole resulting in the absence of $\mathrm{P}$ wave before each QRS complex in the electrocardiogram (ECG). The $\mathrm{P}$ waves are replaced by fibrillatory " $\mathrm{f}$ " waves which vary in size, shape, and timing (caution: atrial fibrillation with complete heart block may present with regular R-R intervals in the ECG).

\section{CLASSIFICATION}

There is no general agreement on the terminology to describe various types of atrial fibrillation. ${ }^{6}$ It may be usually classified as acute and chronic atrial fibrillation. ${ }^{7}$ Chronic atrial fibrillation may be further subclassified as paroxysmal, persistent, and permanent. ${ }^{78}$ When episodes of atrial fibrillation recur and each is self terminating, this is called paroxysmal atrial fibrillation. The term, "persistent" is used when the spontaneous remission of atrial fibrillation does not occur and atrial fibrillation is likely to persist if not cardioverted. It is regarded as permanent when it cannot be reverted to sinus rhythm, even by electrical cardioversion. Most atrial fibrillation usually starts as paroxysmal atrial fibrillation. In this simple but therapeutically useful classification, there may be overlap between acute onset and other types of atrial fibrillation. There are published complex classifications of atrial fibrillation which are beyond the scope of this article. $^{.910}$

\section{MECHANISM}

The mechanism of atrial fibrillation is not fully elucidated. ${ }^{6}$ At least three mechanisms have been shown to be important in its genesis and maintenance.

(1) There may be enhanced automaticity within the small cuffs of left atrial muscle that extend into the pulmonary veins. Multiple ectopic atrial beats arising from these areas may act as the initial triggers for episodes of atrial fibrillation. ${ }^{11}$ In selected patients, paroxysmal atrial fibrillation may be permanently cured by radiofrequency ablation therapy eliminating this triggering focus. ${ }^{12}$

(2) Electrical remodelling of the atria with shortening of the atrial refractory period during episodes of atrial fibrillation helps increase the duration and stability of atrial fibrillation. ${ }^{13}$ This phenomenon is associated with intracellular calcium influx, ${ }^{14}$ and is well described by the phrase "atrial fibrillation begets atrial fibrillation".

(3) In chronic atrial fibrillation, multiple re-entrant waves wander over the surface of atria supported by areas of functional conduction block. Such waves collide and divide and maintain the chaotic electrical state in chronic atrial fibrillation. ${ }^{15}$

\section{CAUSES}

Atrial fibrillation is commonly associated with structural heart disease. The term "lone atrial

Abbreviations: DCC, direct current cardioversion; ECG, electrocardiogram; TOE, transoesophageal echocardiography 
Box 1: Causes of atrial fibrillation

\section{Cardiovascular causes}

- Hypertension.

- Ischaemic heart disease.

- Rheumatic heart disease.

- Cardiomyopathy.

- Pericarditis.

- Congenital heart disease, in particular, atrial septal defect.

- Postoperative cardiac surgery.

- Wolff-Parkinson-White syndrome.

- Hypertrophic cardiomyopathy.

- Sick sinus syndrome.

- Pulmonary embolism.

- Primary pulmonary hypertension.

- Diabetes mellitus.

Coexisting with other cardiac arrhythmias

- Atrioventricular re-entrant tachycardias.

- Atrioventricular nodal re-entrant tachycardias.

- Atrial tachycardia.

Non-cardiovascular causes

- Hyperthyroidism.

- Pneumonia/chronic obstructive airways disease.

- Alcohol binge.

- Postoperative (non-cardiac surgery).

fibrillation" is used when no apparent cause can be identified. A list of clinically important causes of atrial fibrillation is presented in box 1 .

\section{PRINCIPLE OF TREATMENT}

The present treatment of atrial fibrillation is based on four main principles:

(1) Restoration of sinus rhythm.

(2) Rate control.

(3) Maintenance of sinus rhythm.

(4) Prevention of thromboembolism.

However, when a patient with atrial fibrillation is seen in the casualty department or emergency assessment unit, the top priority is to reduce the fast ventricular rate, which can be achieved either by restoration of sinus rhythm or by controlling the rate, depending on the haemodynamic stability of the patient (figs 1 and 2). Heparin should be started on admission.

For some patients with atrial fibrillation who are haemodynamically stable, it is reasonable to wait for 24 hours before attempting to cardiovert as many patients with paroxysmal atrial fibrillation may revert to sinus rhythm spontaneously. ${ }^{16}$

\section{RESTORATION OF SINUS RHYTHM}

Conventionally many clinicians attempt to revert atrial fibrillation to sinus rhythm, although there are few convincing data to support this approach. ${ }^{17}$ Cardioversion of atrial fibrillation is usually achieved with synchronised direct current cardioversion (DCC). All elective DCC is performed under short general anaesthesia to avoid discomfort to the patient. However as a life saving measure for a patient with unstable atrial fibrillation, it is reasonable to perform an urgent DCC under conscious sedation if time permits. Elective DCC under conscious sedation was shown to be safe and not associated with intolerable discomfort to the patients. ${ }^{18}$ Conventionally, monophasic waveform transthoracic direct current defibrillator is used. The sequence of energy commonly used are 100 joules, 200 joules, 300 joules, and 360 joules. It is important to apply firm pressure on the paddles. The success rate of such treatment depends on many factors-for example, duration of atrial fibrillation (the longer the duration, the lesser the chance of success), poor left ventricular function, dilated left atrium, and electrolyte imbalance. The immediate success rate reported in the literature varies from $65 \%$ to $90 \%{ }^{6}$ The Consensus Conference on Atrial Fibrillation of the Royal College of Physicians of Edinburgh recommends cardioversion for atrial fibrillation of less than three months' duration. ${ }^{1}$ It is worthwhile considering an attempt of cardioversion for young patients with atrial fibrillation when the onset is between three and six months. In a patient with atrial fibrillation who is haemodynamically unstable, for example, with hypotension, left ventricular failure, cardiogenic shock, refractory angina and rapid ventricular rate (>200 beats/min), DCC should be urgently undertaken. If delay is anticipated, ventricular rate control could be achieved with intravenous $\beta$-blockers, rate limiting calcium channel blockers, digoxin, or amiodarone. If patient is stable and the onset of the atrial fibrillation is less than 48 hours, pharmacological cardioversion frequently works successfully. Intravenous flecainide is commonly used provided there is no contraindication such as impaired left ventricular function. Efficacy may be as high as $70 \%-80 \%$ in the first few hours after onset of atrial fibrillation and declines thereafter. ${ }^{19}$ If flecainide is contraindicated, intravenous amiodarone is an alternative (fig 1). A single dose of oral flecainide $(300 \mathrm{mg})$ or propafenone $(600 \mathrm{mg})$ were also found to cardiovert atrial fibrillation in $70 \%-80 \%$ at eight hours. ${ }^{20}$ In a patient in whom such a strategy is effective and who is clearly able to recognise the onset of an attack of paroxysmal atrial fibrillation, self administration of one of these drugs may be effective strategy for occasional attacks. This is known as the "pill in the pocket" strategy.

Although ibutilide or dofetilide are not yet available in the UK for clinical use, these new class III antiarrhythmic drugs have been approved for the treatment of atrial fibrillation in the United States. Intravenous ibutilide can be used in patients with atrial fibrillation and ischaemic heart disease. ${ }^{16}$ However, intravenous ibutilide should be avoided in patients with severe left ventricular dysfunction and long QT interval. ${ }^{21}$ Oral dofetilide can also be used in patients with atrial fibrillation and heart failure. ${ }^{6}$

For stable atrial fibrillation of more than 48 hours' duration, elective cardioversion is performed after conventional oral anticoagulation with the international normalised ratio range of 2-3 for three weeks before cardioversion. ${ }^{122}$ However, for urgent cardioversion (both electrical and pharmacological), patient needs to be heparinised to achieve partial thromboplastin time of 1.5 to 2.5 times control before cardioversion. Oral anticoagulation for four weeks is necessary after successful cardioversion if the onset of atrial fibrillation is more than 48 hours. ${ }^{12}$ This treatment strategy is based on the concept that after cardioversion of atrial fibrillation (onset $>48$ hours), "atrial stunning" with impaired left atrial mechanical function occurs and may last for weeks. ${ }^{23}$ This may predispose to intracardiac thrombus formation and thromboembolism. In the next four weeks the international normalised ratio should be maintained between 2 and 3 and an ECG should be performed in four weeks to decide whether to stop warfarin.

An important advance in improving the success rate of electrical cardioversion is the development of the biphasic defibrillator. This device looks very similar to the conventional defibrillator, although the maximum energy which may be selected is 200 joules. Instead of giving a monophasic electrical shock it delivers a biphasic shock, in which the polarity of the shock is automatically reversed during shock delivery. A randomised trial of 165 patients with atrial fibrillation which compared damped sine wave monophasic with rectilinear biphasic transthoracic cardioversion showed an improvement in cardioversion success from $79 \%$ to $94 \% .^{24}$ Despite the lower levels of energy delivered by the biphasic defibrillator, it is likely that the biphasic defibrillator will become widespread in hospitals as there is increasing evidence that it may also be more effective in terminating ventricular fibrillation. The 


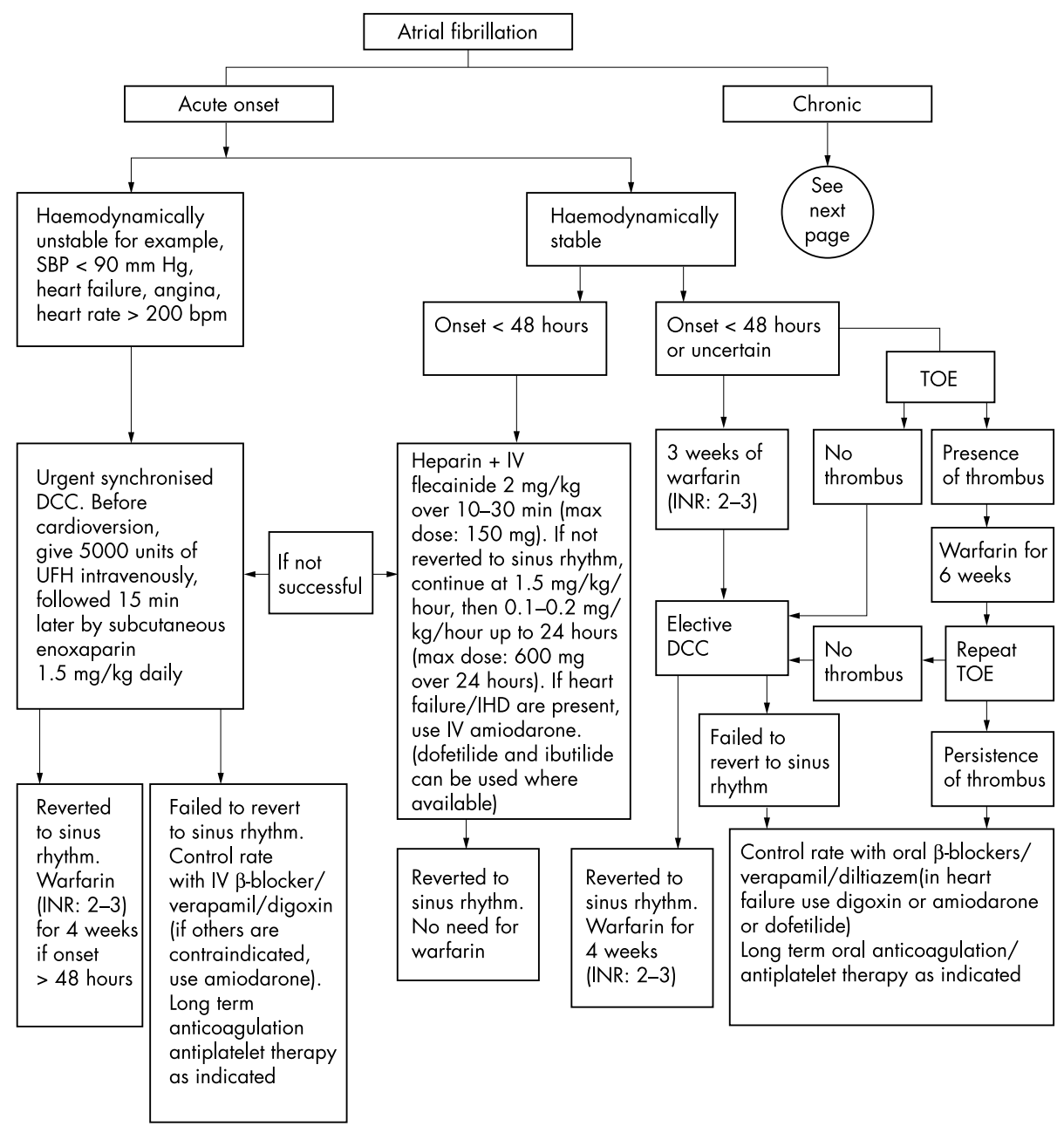

Figure 1 Treatment algorithm for acute onset atrial fibrillation. (Exclude hyperthyroidism and electrolyte imbalance on admission.) DCC direct current cardioversion; IHD ischaemic heart disease; INR, international normalised ratio; IV, intravenous; SBP, systolic blood pressure; TOE, transoesophageal echocardiography; UFH, unfractionated heparin.

biphasic defibrillator is available for clinical use in the UK and is likely to become the standard defibrillator in the near future..$^{25}$

The success rate of electrical cardioversion of atrial fibrillation and maintenance of sinus rhythm can be increased by treatment with certain antiarrhythmic drugs before and after cardioversion. ${ }^{1}$ Commonly used antiarrhythmic drugs include those of Vaughan Williams class Ia, class Ic, and class III-namely, flecainide, propafenone, procainamide, quinidine, and amiodarone. Oral dofetilide may be used where available. Oral et al have suggested that the success rate of DCC could improve up to $100 \%$ if DCC is performed after pretreatment with intravenous ibutilide. ${ }^{21}$ In their trial, DCC was performed after pretreatment with $1 \mathrm{mg}$ of ibutilide infused over 10 minutes. However, the drug increased risk of ventricular fibrillation in patients with severe left ventricular dysfunction and long QT interval. It is also possible to achieve cardioversion by delivering the shock energy internally. The procedure is performed using a special defibrillator that connects to a transvenous electrode system which has electrodes in the right atrium and pulmonary artery. Despite the lower energy level used in this system the local delivery of energy to the heart achieves a high success rate. Out of 20 patients with chronic atrial fibrillation in whom conventional transthoracic (external) cardioversion failed, low energy transvenous (internal) cardioversion was successful in $75 \%{ }^{26}$ In another large multicentre trial of 500 patients, the transvenous cardioversion was successful in restoring sinus rhythm in $>90 \%$ of cases (which included $57 \%$ of failed external cardioversion). ${ }^{27}$ Low energy internal cardioversion seems to be safe and effective means of terminating atrial fibrillation that has been resistant to conventional cardioversion. Because it is invasive and requires quite expensive disposable catheter, it should be considered only if biphasic cardioversion has failed. Referral to a specialist centre will be required.

\section{TRANSOESOPHAGEAL ECHOCARDIOGRAPHY GUIDED CARDIOVERSION}

The serious complication of cardioversion for atrial fibrillation is thromboembolism in 5\%-7\% without anticoagulation and $1 \%-2 \%$ after conventional anticoagulation. ${ }^{1}$ However, from the data of 1164 patients who underwent transoesophageal echocardiography (TOE) guided DCC and who had atrial fibrillation or atrial flutter, Grimm reported that TOE guided DCC was associated with very low thromboembolic complications of $<0.1 \%{ }^{28}$ This strategy involves performing TOE after intravenous unfractionated heparin for $1-5$ days (keeping the partial thromboplastin time between 1.5 and 2.5 times control) or warfarin therapy for at least five days with an international normalised ratio of $2-3 .{ }^{28}$ When atrial thrombus is excluded, DCC is performed. If a thrombus is detected, DCC should be postponed and the patient should be warfarinised for six weeks or longer. ${ }^{6}$ If the thrombus resolves, DCC can then be performed; if not, it is generally abandoned. ${ }^{6}$ In a trial of TOE guided cardioversion for 242 patients with atrial fibrillation and atrial flutter, two thirds of patients with atrial fibrillation and atrial flutter of $>48$ hours' duration were successfully cardioverted without increasing risk of thromboembolism. ${ }^{29}$ The authors also showed that the reduction in time to DCC was associated with better maintenance of sinus rhythm at one month, in comparison with those patients who underwent conventional oral anticoagulation before cardioversion. 


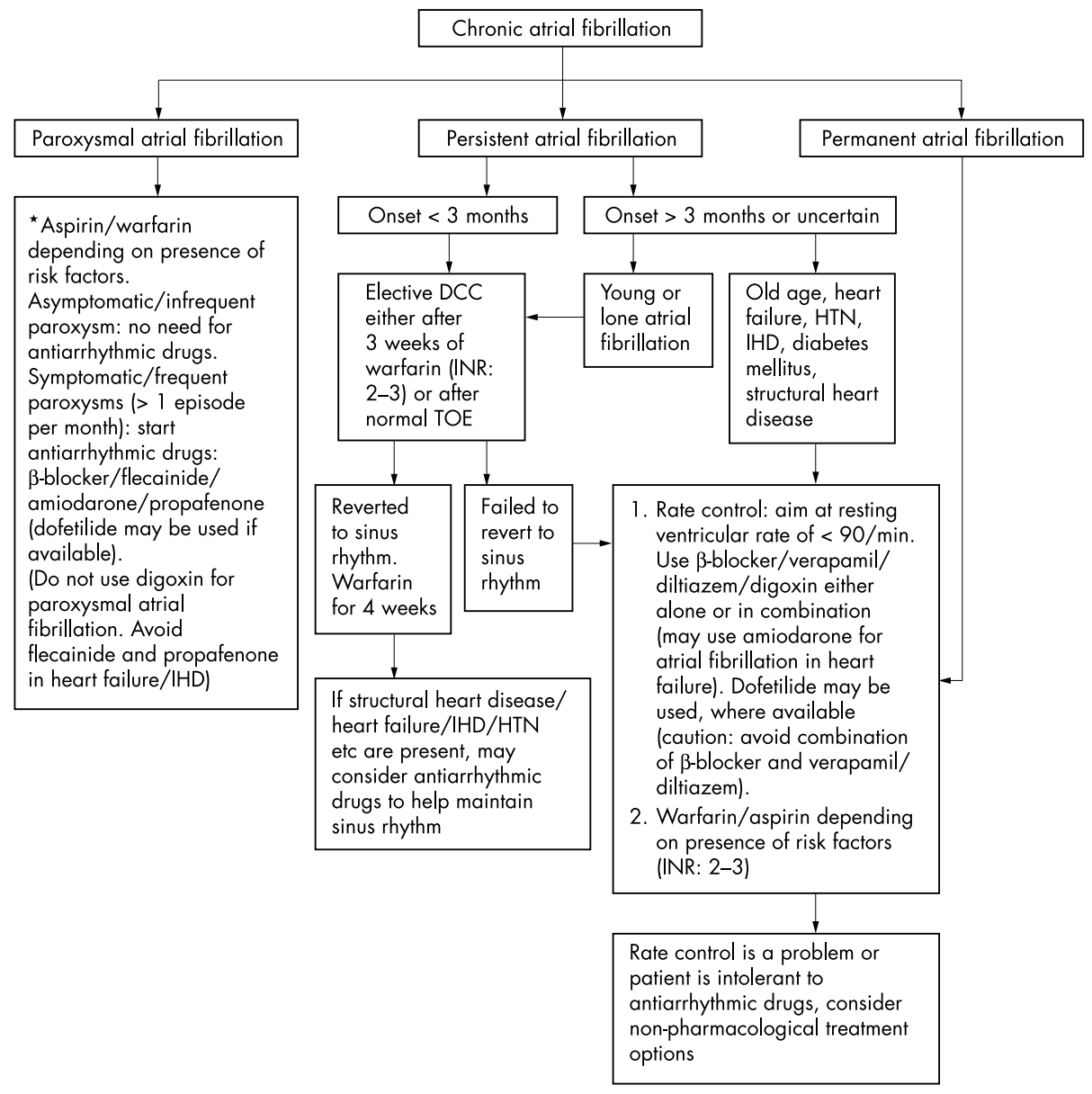

Figure 2 Treatment algorithm for chronic atrial fibrillation. DCC, direct current cardioversion; HTN, hypertension; IHD, ischaemic heart disease; INR, international normalised ratio; TOE, transoesophageal echocardiography. *If intolerant/allergic to aspirin, use clopidogrel/modified release dipyridamole.

\section{risk factors.}

Asymptomatic/infrequent

aroxysm: no need for Symptomatic/frequen $\beta$-blocker/flecainide/ fibrillation. Avoid flecainide and propafenone in heart failure/IHD

The ACUTE trial which randomised 1222 patients with atrial fibrillation and atrial flutter (onset $>48$ hours) to TOE guided DCC and conventional DCC showed that the success rate of DCC was higher in patients who underwent TOE guided DCC $(p=0.03) .{ }^{30}$ Although the embolic stroke was similar on both sides, the haemorrhagic complication was significantly higher in patients treated with conventional DCC. However, at eight weeks, there was no difference in maintenance of sinus rhythm or death or functional status between the two groups. TOE guided DCC for atrial fibrillation can be used as an effective and safe alternative to the conventional DCC in centres where TOE service is available.

\section{RATE CONTROL}

In case of failure of rhythm control, it is necessary to control the resting ventricular rate at $<90$ beats/min, which is about $20 \%$ higher than the heart rate while in sinus rhythm..$^{131}$ The heart rate should not exceed 180 beats/min on exercise. ${ }^{1}$ The rate control can be achieved either pharmacologically or by non-pharmacological means. The antiarrhythmic drugs which increase refractory period of the atrioventricular node and delay atrioventricular conduction are preferred. The commonly used drugs are $\beta$-blockers, verapamil, diltiazem, and digoxin. Although digoxin remains the first choice in presence of heart failure, it should be avoided in patients with atrial fibrillation associated with Wolff-Parkinson-White syndrome and hypertrophic obstructive cardiomyopathy. It is relatively ineffective in paroxysmal atrial fibrillation as it fails to prevent recurrence or control rate. ${ }^{1}$ Calcium channel blockers are to be avoided in heart failure except when the heart failure is secondary to the fast atrial fibrillation. Although amiodarone is not considered in a first line drug for rate control because of its adverse effect, it has a place in treating patients with atrial fibrillation with severe heart failure.
The rate control has some disadvantages. The atrial contribution to the cardiac output is permanently lost. Atrial fibrillation may cause progressive dilatation of left atrium. ${ }^{17}$ Poorly controlled permanent atrial fibrillation may result in so called "tachycardia induced cardiomyopathy" ${ }^{32}$ The other disadvantage is the need for long term anticoagulation therapy or antiplatelet therapy.

Elderly patients with atrial fibrillation may have concomitant atrioventricular nodal disease which slows the atrioventricular conduction. As ventricular rate is usually reasonable in such patients, rate limiting drugs are usually not required..$^{33}$

\section{RHYTHM CONTROL VERSUS RATE CONTROL}

The advantages of rhythm control are restoration of "normal rhythm", symptom improvement, and freedom from long term anticoagulation or antiplatelet therapy. Even for patients with atrial fibrillation with controlled ventricular rate, the left ventricular function improved on restoration of sinus rhythm with cardioversion. ${ }^{34}$ After cardioversion, relatively few patients remain in sinus rhythm. In a trial of 236 patients with atrial fibrillation who underwent serial electrical cardioversions with prophylactic antiarrhythmic drug therapy, only $42 \%$ and $27 \%$ of successfully cardioverted patients remained in sinus rhythm after one year and four years. ${ }^{35}$ Although restoration of left atrial contribution to stroke volume could be important in a failing left ventricle, the influence of rhythm control on the survival of patients with atrial fibrillation is not clear. ${ }^{17}$ The result of the Pharmacological Intervention in Atrial Fibrillation trial, which randomised 252 patients with atrial fibrillation to rhythm or rate control, did not show any significant difference in symptom improvement between the two treatment strategies, although exercise tolerance was better with rhythm control at the cost of more hospitalisation. ${ }^{36}$ 
The three larger unpublished trials (AFFIRM, RACE, STAF) comparing rhythm control with rate control $^{17}$ are likely to resolve the issue. The AFFIRM (Atrial Fibrillation Follow-up Investigation of Rhythm Management) trial enrolled 4060 patients with an average follow up of 3.5 years. ${ }^{37}{ }^{38}$ In the 51 st Annual Scientific Session of the American College of Cardiology (reported by Sue Hughes and Lisa Nainggolan ${ }^{38}$ ), Dr George Wyse, the chief investigator presented the result of the AFFIRM trial, which did not show significant difference in mortality, functional status, quality of life, or risk of ischaemic stroke between the two groups.

The RACE (Rate Control versus Electrical Cardioversion for Persistent Atrial Fibrillation) trial which randomised 522 patients with atrial fibrillation to rhythm control or rate control did not show any significant difference in mortality at the end of three years of follow up. ${ }^{38}$ The STAF (Strategies of Treatment of Atrial Fibrillation) trial aiming at randomising more than 2000 patients started in 1997 has not yet been published.

In an individual patient a decision on whether to pursue a strategy of rhythm control or rate control will need to take into account the evidence above, the effectiveness of treatments already tried, the risks of anticoagulation in that individual, the patient's tolerance of atrial fibrillation whether paroxysmal or established, and the patient's own viewpoint. Some patients are very aware of the presence of atrial fibrillation and wish to pursue a vigorous strategy of sinus rhythm maintenance. In others atrial fibrillation may be well tolerated. The data emerging from the trials suggest that in these patients a rate control strategy may be pursued without adverse effects.

\section{MAINTENANCE OF SINUS RHYTHM}

The relapse rate after initial successful cardioversion is high at $25 \%-50 \%$ at one month and $70 \%-90 \%$ at one year. ${ }^{1}$ Prophylactic antiarrhythmic drugs are usually required for a significant number of patients with atrial fibrillation after successful cardioversion. Beta-blockers or Vaughan Williams class Ic drugs-for example, flecainide or propafenone-are considered as first line drugs. ${ }^{1}$ Paroxysmal atrial fibrillation may be considered frequent if the episode is more than one per month. ${ }^{39}$ Such patients may require antiarrhythmic drug therapy. Sotalol may be considered as the initial antiarrhythmic drug for patients with atrial fibrillation and ischaemic heart disease. ${ }^{39}$ When class I antiarrhythmic drugs are contraindicated as in significant ischaemic heart disease or left ventricular dysfunction, amiodarone is an alternative. In such patients, oral dofetilide may also be used to maintain sinus rhythm after cardioversion in countries where it is available. 1639

\section{PREVENTION OF THROMBOEMBOLISM}

Chronic atrial fibrillation is associated with embolic stroke in around 5\% per year. ${ }^{11}$ Although there is lack of unanimity over the relative benefit of anticoagulation and antiplatelet treatment for patients with atrial fibrillation at low to moderate risk of thromboembolism, there is consensus on the use of anticoagulation for those patients who are at high risk of thromboembolism. The guideline of the British Committee for Standards in Haematology recommends warfarin as the first line antithrombotic treatment for all patients with atrial fibrillation who have at least one risk factor for thromboembolism..$^{40}$ The important risk factors are presented in the box $2 .^{14}$

Those patients between 65 and 75 years of age are at moderate risk and may be considered for warfarin therapy. Although the analysis of pooled data of the Atrial Fibrillation, Aspirin, Anticoagulation Study (AFSAK), the European Atrial Fibrillation Trial (EAFT), and the Stroke Prevention in Atrial Fibrillation 1 Study (SPAF 1) showed a $21 \%$ relative risk
Box 2: Risk factors for ischaemic stroke associated with atrial fibrillation

- $75+$ years of age

- Previous episodes of cerebral embolism.

- Congestive heart failure.

- Left ventricular dysfunction by echocardiography.

- Hypertension.

- Diabetes mellitus

- Coronary artery disease.

- Mitral or aortic valve disease.

- Cardiac valve repair.

- Post-valvuloplasty.

reduction of ischaemic stroke with aspirin therapy which was not statistical significant $(p=0.05),{ }^{42}$ aspirin $(75-300 \mathrm{mg} /$ day $)$ may be considered if warfarin is contraindicated. When patients are intolerant or allergic to aspirin, it is reasonable to give clopidogrel or modified release dipyridamole. ${ }^{43}$ Low risk patients, for example those $<65$ years of age without any risk factor for thromboembolism, may be left alone or be given aspirin if there is no contraindication to aspirin. Patients at low risk or moderate risk (but not on warfarin) should be periodically checked for any development of high risk when warfarin should be promptly initiated.

Patients with recurrent attacks of paroxysmal atrial fibrillation at high risk of thromboembolism should receive appropriate antithrombotic therapy. ${ }^{44}$

\section{NON-PHARMACOLOGICAL APPROACHES FOR TREATMENT OF ATRIAL FIBRILLATION}

When drug treatment fails to achieve either rhythm control or rate control, the various non-pharmacological approaches to achieve rhythm or rate control should be considered for symptomatic atrial fibrillation.

\section{Ablate and pace to control heart rate}

This technique has been more widely practised than many other non-pharmacological methods for treatment of atrial fibrillation. In this method, the atrioventricular junction is completely ablated by catheter based radiofrequency energy along with permanent pacing. The atrial systole is not restored and the patient is likely to require long term anticoagulation. The technique was shown to improve symptoms, quality of life, exercise capacity, and left ventricular function and also reduce the number of hospital readmission. ${ }^{45}$ In another trial, which enrolled 350 patients with drug refractory atrial fibrillation, atrioventricular nodal ablation with radiofrequency energy was performed with either ventricular pacing (55\% of patients) or a dual chamber pacing (45\% of patients). ${ }^{46}$ Although it was not a randomised controlled trial, the trial showed that the survival of patients with atrial fibrillation without structural heart disease who underwent the ablate and pace strategy was similar to that of the population control at the end of a mean follow up of $36+/-26$ months. In a multicentre but non-randomised trial consisting of 156 patients with drug refractory atrial fibrillation, atrioventricular junctional ablation and pacing was shown to significantly improve quality of life and left ventricular ejection fractions. ${ }^{47}$ There was also a trend towards improvement of exercise capacity after the procedure, although it did not reach statistical significance.

\section{Conventional pacing to prevent atrial fibrillation}

The randomised controlled trial by Andersen et al, which enrolled 225 patients, showed that atrial pacing in the treatment of sick sinus syndrome was superior to ventricular pacing (VVI) in preventing development of atrial fibrillation at 
the end of mean follow up of 40 months ( $14 \% v 23 \%) .{ }^{48}$ Moreover, the thromboembolic complication was lower in atrially paced patients $(\mathrm{p}<0.01)$. There was, however, no significant difference in survival.

Permanent pacing may be required for patients with atrial fibrillation who have symptomatic bradycardia as a result of antiarrhythmic drug treatment or high degree atrioventricular block.

\section{Ablation to prevent or stop atrial fibrillation}

Atrial ablation can be achieved either by catheter based technique or open heart surgery. In the catheter based technique, multiple linear ablations are made in the right or/and left atria by radiofrequency energy. The success rate of left atrial ablation is higher than the right atrial ablation. ${ }^{39}$ More recently foci of atrial tissue with abnormal automaticity triggering atrial fibrillation were detected in abnormal sites mainly within the pulmonary veins (>90\%) which were considered suitable for catheter ablation. ${ }^{11}{ }^{45}$ Chen et al showed that, of all ectopic foci in the pulmonary veins which were demonstrated to initiate atrial fibrillation, radiofrequency ablation was successful in eliminating $95 \%$ of the foci. ${ }^{49}$ Moreover, $86 \%$ of the 79 patients studied remained in sinus rhythm at the end of $6+/-2$ months of follow up. Although a few potential complications namely pulmonary vein stenosis, systemic embolism and pericardial effusion were reported, radiofrequency ablation of atrial fibrillation foci within pulmonary veins seems promising.

\section{Implantable atrial defibrillator}

The principle of the implantable atrial defibrillator was derived from that of the implantable cardioverter defibrillator for the treatment of ventricular tachyarrhythmia. It involves putting in transvenous leads in the right atrium and also in coronary sinus which are connected to the defibrillator located subcutaneously in the infraclavicular region. The device is usually triggered manually at a preset delivery energy varying from 0.1 to 10.0 joules. However, a majority of patients reported significant discomfort with energy $>2.0$ joules. $^{50}$ The Metrix system trial which enrolled 51 selective patients with atrial fibrillation showed success rate of $86 \%$ without significant induction of ventricular tachyarrhythmias or unsynchronised delivery of energy or thromboembolism..$^{51}$ However, the early recurrence rate was high at $27 \%$. It is considered that by terminating atrial fibrillation promptly with the implantable atrial defibrillator that it will decrease the duration of atrial fibrillation and thereby may help reduce/prevent atrial fibrillation induced atrial remodelling..$^{52}$ Although patient tolerability is usually poor and the need for long term anticoagulation is not clear, an implantable atrial defibrillator as a back up device in addition to pharmacological therapy is a therapeutic option for difficult atrial fibrillation.

\section{Surgical approach for treatment of atrial fibrillation}

Maintenance of atrial fibrillation requires a certain critical mass of atrial tissue, to allow the propagation of multiple waves of electrical depolarisation. A surgical procedure called the Maze operation uses multiple incisions in the atria to create linear scars which separate the atrial myocardium into small strips. ${ }^{53}$ The strips remain connected at their ends, allowing normal depolarisation and contraction with sinus rhythm. However the scars between the strips act as barriers to the maintenance of the multiple electrical wavelets which are required for persistent atrial fibrillation (fig 3).

This operation has been refined over the years and the latest version (Maze III procedure) achieves maintenance of sinus rhythm in $>93 \%$ of patients three months after the procedure. ${ }^{55}$ However, because of requirement for open chest operation and extracorporeal circulation, this surgical technique is limited to a small group of patients with paroxysmal atrial fibrillation who undergo open heart surgery for other reasons. ${ }^{45}$

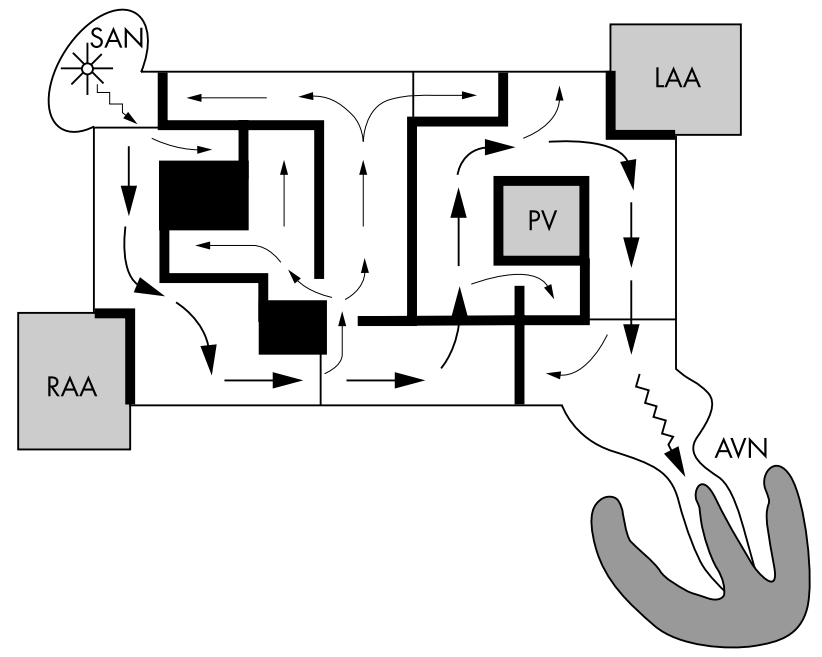

Figure 3 Maze procedure for atrial fibrillation. Both atrial appendages are excised and the pulmonary veins (PV) are isolated. Appropriately placed atrial incisions not only interrupt the conduction routes of the most common re-entrant circuits, but they also direct the sinus impulse from the sinoatrial node (SAN) to the atrioventricular node (AVN) along a specified route. The entire atrial myocardium (except for the atrial appendages and pulmonary veins) is electrically activated by providing for multiple blind alleys off the main conduction route between the sinoatrial node and the atrioventricular node, thereby preserving atrial transport function postoperatively. ${ }^{54}$ LAA, left atrial appendage; RAA, right atrial appendage. (Reproduced with kind permission of the WB Saunders Company.)

\section{SUMMARY}

Reduction of fast ventricular rate along with antithrombotic therapy should be the first step in the treatment of atrial fibrillation. Long term rhythm or rate control should be assessed on an individual basis, considering the safety of patients, presence or absence of substrate to prevent or reduce the chance of maintenance of sinus rhythm after cardioversion, patient tolerability of the antiarrhythmic drugs, and risk of long term anticoagulation. Although evidence is emerging to indicate that there is no significant difference in mortality, functional status, quality of life, or risk of ischaemic stroke between rhythm control and rate control of atrial fibrillation, it seems reasonable to try to restore sinus rhythm for a subgroup of patients with atrial fibrillation-for example, patients with heart failure when restoration of left atrial contribution to stroke volume may help improve left ventricular function. When rhythm control strategy is pursued the standard mode of restoration of sinus rhythm is DCC, although pharmacological cardioversion is effective frequently in acute onset atrial fibrillation. The success rate of DCC could further improve by treatment with antiarrhythmic drugs before cardioversion. A low energy biphasic waveform defibrillator is superior to the conventional monophasic wave defibrillator and is likely to become the standard defibrillator in the near future. Internal cardioversion may also be considered after failed transthoracic cardioversion if such facility is available. TOE guided DCC of atrial fibrillation may be used to reduce the period of anticoagulation before cardioversion without increasing the risk of thromboembolism. When pharmacological rhythm or rate control fail, symptomatic patients with symptomatic atrial fibrillation should be given the newer non-pharmacological treatment options.

Authors' affiliations

S K S Lairikyengbam, A G Davies, Bronglais General Hospital, Aberystwyth

M H Anderson, Regional Cardiac Centre, Morriston Hospital, Swansea 


\section{REFERENCES}

Consensus Conference on Atrial Fibrillation in Hospital and General Practice. Final consensus statement. Proc R Coll Physicians Edinb 1999;29(suppl 6):2-3.

2 Bialy D, Lehmann MH, Schumacjer DN, et al. Hospitalisation from arrhythmias in the United States: importance of atrial fibrillation (abstract). J Am Coll Cardiol 1992;19:41A.

3 Benjamin EJ, Wolf PA, D'Agostino RB, et al. Impact of atrial fibrillation on the risk of death: the Framingham Heart Study. Circulation 1998; 98:946-52

4 Wolf PA, Abbott RD, Kannel WB. Atrial fibrillation as an independent risk factor for stroke: the Framingham Study. Stroke 1991;22:983-8.

5 Lin HJ, Wolf PA, Kelly-Hayes M, et al. Storke severity in atrial fibrillation. The Framingham Study. Stroke 1996;27:1760-4.

6 Levy S, Breithardt G, Campbell RWF, et al. Working Group Report. Atrial fibrillation: current knowledge and recommendation for management. Eur Heart J 1998;19:1294-320.

7 Sopher SM, Camm AJ. Theory for atrial fibrillation: control of the ventricular response and prevention of recurrence. Coron Artery Dis 1995:6:106-14.

8 Waktare JEP, Camm AJ. How do we maintain sinus rhythm in paroxysmal atrial fibrillation. Consensus statement on atrial fibrillation in hospital and general practice. Proc R Coll Physicians Edinb 1999;29:5-12.

9 Gallagher MM, Camm AJ. Classification of atrial fibrillation. PACE 1997;20:1603-5

10 Levy S. Classification system of atrial fibrillation. Curr Opin Cardiol 2000; 15:54-7

11 Pelosi F Jr, Morady F. Evaluation and management of atrial fibrillation. Med Clin North Am 2001:85:225-44.

12 Jais $\mathbf{P}$, Haissaguerre $M$, Shah DC, et al. A focal source of atrial fibrillation treated by discrete radiofrequency ablation. Circulation 1997; $95: 572-6$

13 Daoud EG, Bogun F, Goyal R, et al. Effect of atrial fibrillation on atria refractoriness in human. Circulation 1996;94:1600-6.

14 Ausma J, Dispersyn GD, Duimel $\mathrm{H}$, et al. Change in ultrastructural calcium distribution in goat atria during atrial fibrillation. J Mol Cell Cardiol 2000:32:355-64.

15 Moe GK, Rheinboldt WC, Abildskov JA. Computer model for atrial fibrillation. Am Heart J 1964;67:200-20

16 Reiffee JA, Camm AJ, Haffajee Cl, et al. International consensus roundtable on atrial fibrillation. Cardiology Review 2000;17(suppl): 119.

17 Carlsson J, Neuzner J, Rosenberg YD. Therapy of atrial fibrillation: rhythm control versus rate control. PACE 2000;23:891-903.

18 Raipancholia R, Sentinella L, Lynch M. Role of conscious sedation for external cardioversion. Heart 2001;86:571-2.

19 Reisinger JJ, Gattere E, Heinze G, et al. Prospective comparison of flecainide versus sotalol for immediate cardioversion of atrial fibrillation. Am J Cardiol 1998:81:1450-4.

20 Capucci A, Boriani G, Botto GL, et al. Conversion of recent onset atrial fibrillation to sinus rhythm by a single oral loading dose of propafenone or flecainide. Am J Cardiol 1994;74:503-5.

21 Oral H, Souza JJ, Michaud GF, et al. Facilitating transthoracic cardioversion of atrial fibrillation with ibutilide pretreatment. N Engl J Med 1999;340: 1849-54.

22 Anonymous. Proceeding of the American College of Chest Physicians 5th Consensus on Antithrombotic Therapy, 1998. Chest 1998;114:439S-769S.

23 Harjai KJ, Mobarek SK, Cheirif J, et al. Clinical variables affecting recovery of left atrial mechanical function after cardioversion for atrial fibrillation. J Am Coll Cardiol 1994;74:503-5.

24 Mittal S, Ayati S, Stein KM, et al. Transthoracic cardioversion of atrial fibrillation: comparison of rectilinear biphasic versus damped sine wave monophasic shocks. Circulation 2000;101:1282-7.

25 Trohman RG, Parrillo JE. Direct current cardioversion: indications, techniques and recent advances. Crit Care Med 2000;28:N170-3.

26 Taramasco V, Socas A, Ricard P, et al. Internal low-energy cardioversion: a therapeutic option for restoring sinus rhythm in chronic atrial fibrillation after failure of external cardioversion. Europace 1999;1:179-82.

27 Andrahgetti A, Scalese M. Safety and efficacy of low-energy cardioversion of 500 patients using two different techniques. Europace 2001;3:4-9.

28 Grimm RA. Transoesophageal echocardiography-guided cardiversion of atrial fibrillation. Echocardiography 2000;17:383-92.
29 Roijer A, Eskilsson J, Olsson B. Transoesophageal echocardiography-guided cardioversion of atrial fibrillation or flutter. A selection of low-risk group for immediate cardioversion. Eur Heart J 2000;20:837-47

30 Klein AL, Grimm RA, Murray RD, et al. Assessment of Cardioversion Using Transesophageal Echocardiography Investigators. Use of transesophageal echocardiography to guide cardioversion in patients with atrial fibrillation. N Engl J Med 2001;344:1411-20.

31 Rawles JM. What is meant by a "controlled" ventricular rate in atrial fibrillation. Br Heart J 1990:63:157-61.

32 Fenelon G, Wijns W, Andries E, et al. Tachycardiomyopathy: mechanism and clinical implications. PACE 1996;19:95-106.

33 Zipes D. Specific arrhythmias: diagnosis and treatment in Braunwald's heart disease. In: Braunwald E, ed. A textbook of cardiovascular medicine. 4th Ed. Philadelphia: W B Saunders, 1992.

34 Kubec G, Malowany L. Functional capacity of patients with atrial fibrillation and controlled heart rate before and after cardioversion. Can J Cardiol 1992:8:941-6.

35 Van Gelder IC, Crijns HJGM, Tieleman RG, et al. Chronic atrial fibrillation. Success of serial cardioversion therapy and safety of oral anticoagulation. Arch Intern Med 1996;156:2585-92.

36 Hohnloser SH, Kuck KH, Lilienthal J. Rhythm or rate control in atrial fibrillation. Pharmacological Intervention in Atrial Fibrillation (PIAF): a randomised trial. Lancet 2000:356:1789-94.

37 Planning and Steering Committee of the AFFIRM Study for the NHLBI-AFFIRM Investigation. Atrial fibrillation follow-up investigation of rhythm management-design. Am J Cardiol 1997;79: 1 198-202.

38 Hughes S, Nainggolan L. News from the 51 st Annual Scientific Session of the American College of Cardiology. Br J Cardiol 2002;9:206.

39 Fuster V, Ryden LE, Asinger RW, et al. ACC/AHA/ESC guidelines for the management of atrial fibrillation: executive summary. J Am Coll Cardiol 2001;38:1231-65.

40 Haemostasis and Thrombosis Task Force. Prepared for the British Committee for Standards in Haematology (BCSH). Guidelines on oral anticoagulation: third edition. Br J Haematol 1998;101:374-87.

41 Barwolf CG. Anticoagulation in valvular heart disease and management during non-cardiac surgery. Heart 2000:84:567-72

42 Atrial Fibrillation Investigators. The efficacy of aspirin in patients with atrial fibrillation-analysis of pooled data from 3 randomised trials. Arch Intern Med 1997; 157:1237-40.

43 Lairikyengbam SKS, Davies AG, Jones PD. Implementation of antithrombotic management in atrial fibrillation-a correspondence. Postgrad Med J 200 1;77:488.

44 Atrial Fibrillation Investigators. Risk factors for stroke and efficacy of antithrombotic therapy in atrial fibrillation. Arch Intern Med 1994:154:1449-57.

45 Vardas PE. Non-pharmacological treatment of atrial fibrillation: a heretic's appraisal. PACE 2000;23:395-401.

46 Ozcan C, Jahangir A, Friedman PA, et al. Long-term survival after ablation of the atrioventricular node and implantation of a permanent pacemaker in patients with atrial fibrillation. N Engl J Med pacemaker in patients

47 Wood MA, Kay GN, Ellenbogen KA for the APT Investigators. The North American experience with the Ablate and Pace Trial (APT) for medically refractory atrial fibrillation. Europace 1999;1:22-5

48 Andersen HR, Thensen L, Bagger J, et al. Prospective randomised trial of atrial versus ventricular pacing in sick sinus syndrome. Lancet 1994:344:1923-8

49 Chen SA, Hsieh MH, Tai CF, et al. Initiation of atrial fibrillation by ectopic beats originating from the pulmonary veins: electrophysiological characteristics, pharmacological responses, and effects of radiofrequency ablation. Circulation 1999:100:1879-86.

50 Jung J, Heisel A, Fries $R$, et al. Tolerability of internal low-energy shock strengths currently needed for endocardial atrial cardioversion. Am J Cardiol 1997:80: 1489-90.

51 Wellen HJ, Lan CP, Luderitz B, et al. Atrioverter: an implantable device for the treatment of atrial fibrillation. Circulation 1998;98:1651-6.

52 Wijffels MCEF, Kirchhof CJHJ, Dorland RD, et al. Atrial fibrillation begets atrial fibrillation. A study in awake chronically instructed goats. Circulation 1995:92:1954-68.

53 Cox JL, Schuessler RB, Cain ME, et al. Surgery for atrial fibrillation. Semin Thorac Cardiovasc Surg 1989;1:67-73.

54 Cox JL, Schuessler RB, D'Agostino HJ Jr, et al. The surgical treatment of atrial fibrillation. III. Development of a definitive surgical procedure. J Thorac Cardiovasc Surg 1991;101:569-83.

55 Cox JL, Schuessler RB, Lappas DG, et al. An 81/2-year experience with surgery for atrial fibrillation. Ann Surg 1996;224:267-75. 